\title{
Are Angiotensin-Converting Enzyme Inhibitors and Angiotensin Receptor Blockers Especially Useful for Cardiovascular Protection?
}

\author{
Hean Teik Ong, FRCP, FACC, FESC
}

Purpose: This article seeks to objectively review the clinical trial evidence to determine whether angiotensin-converting enzyme inhibitors (ACEIs) and angiotensin-receptor blockers (ARBs) have special cardiovascular protective effects.

Methods: An objective review of the clinical trial evidence.

Results: Clinical trials in hypertensive patients comparing ACEI and ARB with other drugs generally showed no difference in the primary cardiovascular outcome (United Kingdom Prospective Diabetes Study Group, Captopril Prevention Project, Swedish Trial in Old Patients with Hypertension 2, Japan Multicenter Investigation for Cardiovascular Diseases-B Randomized Trial, Antihypertensive and LipidLowering Treatment to Prevent Heart Attack Trial, Second Australian National Blood Pressure Study Group, Valsartan Antihypertensive Long-Term Use Evaluation). Where the primary, or major secondary, cardiovascular end-point favors one of the treatment arms, it was always the arm with the lower achieved blood pressure that saw the better clinical result as in Losartan Intervention For Endpoint Reduction in Hypertension Study, Captopril Prevention Project, Antihypertensive and Lipid-Lowering Treatment to Prevent Heart Attack Trial, and Valsartan Antihypertensive Long-Term Use Evaluation. Trials comparing ACEI or ARB against placebo in patients at high risk of cardiovascular events have not showed a consistent result; cardiovascular outcomes were reduced in Heart Outcomes Prevention Evaluation, European Trial on Reduction of Cardiac Events with Perindopril in Stable Coronary Artery Disease, and the Jikei Heart Study, but were not significantly reduced in Perindopril Protection Against Recurrent Stroke Study, Comparison of Arnlodipine vs Enalapril to Limit Occurrences of Thrombosis Trial, Prevention of Events with ACEIs Trial, Telmisartan Randomized Assessment Study in ACE-Intolerant Subjects with Cardiovascular Disease Trial, and Prevention Regimen for Effectively Avoiding Second Strokes Trial. In the Ongoing Telmisartan Alone and in Combination with Ramipril Global Endpoint Trial, combining ACEIs with ARBs in high-risk patients did not reduce cardiovascular or renal outcomes compared with ACEI monotherapy alone. This absence of a reduction in cardiovascular outcome from the ACEI and ARB combination arm is further evidence suggesting that these drugs do not have any special cardiovascular protective effect. This objective review thus shows that the rennin-angiotensin antagonists do not have special cardiovascular protective properties.

Conclusion: The key to reducing cardiovascular outcome is to appropriately control blood pressure as well as to treat all other coronary risk factors. (J Am Board Fam Med 2009;22:686-697.)

Angiotensin-converting enzyme inhibitors (ACEIs) and angiotensin-receptor blockers (ARBs) produce

This article was externally peer reviewed.

Submitted 25 April 2009; revised 24 July 2009; accepted 28 July 2009.

From the HT Ong Heart Clinic, Penang, Malaysia.

Funding: none.

Conflict of interest: none declared.

Corresponding author: Dr Hean Teik Ong, HT Ong Heart Clinic, 251-C Burma Road, Penang 10350, Malaysia (E-mail: htyl@streamyx.com). no metabolic adverse consequences and are said to have special cardiovascular protective effects in highrisk patients. ${ }^{1-4}$ However, recent clinical trials have shown that practical reality may be different from theoretical benefit, and improvement of metabolic parameters did not produce the expected reduction of clinical outcomes in diabetes and dyslipidemia. ${ }^{5,6}$ This article seeks to objectively review the trial evidence to determine whether ACEIs and ARBs do indeed have special cardiovascular protective properties. 


\section{Method}

A PubMed search was conducted using the keywords "hypertension," "high risk," "coronary disease," "cardiovascular outcome," "ACEIs," "ARBs," and "randomized, controlled, trials." The results were supplemented by references of the retrieved articles, as well as the Seventh Report of the Joint National Committee on Prevention, Detection, Evaluation, and Treatment of High Blood Pressure; the World Health Organization; the British Hypertension Society/National Institute for Health and Clinical Excellence; and the European hypertension guidelines. Trials selected were prospective, randomized (level 1 study quality) trials recruiting more than 500 patients, had follow-up of more than 2 years, and had clinical primary endpoints. Two types of trials were indentified: those in hypertensive patients where ACEIs/ ARBs were compared with other antihypertensive drugs and those in which ACEIs/ARBs were compared with placebo among patients at high cardiovascular risk.

\section{Do ACEIs Have Special Cardiovascular Protective Effects?}

Trials assessing the value of ACEIs in reducing cardiovascular disease can be divided into 2 types: (1) those comparing ACEIs with other drugs in hypertensive patients, and (2) those comparing ACEIs with placebo among patients at high cardiovascular risk who actually have a combination of diabetes, hypertension, hyperlipidemia, and preexisting clinical athromatous disease.

Clinical trials comparing ACEIs with other antihypertensive drugs generally showed no difference in cardiovascular outcome (Table 1). In the United Kingdom Prospective Diabetes Study Group, 758 hypertensive diabetics had their blood pressure (BP) tightly controlled with either captopril or atenolol. ${ }^{7}$ Both treatment arms had similar $\mathrm{BP}$ reduction and after 9 years there was no difference in the primary outcome of diabetic-related clinical events (relative risk [RR] for captopril, 1.10; 95\% CI, 0.86-1.41); stroke (RR, 1.12; 95\% CI, 0.59-2.12); myocardial infarction (MI) (RR, 1.20; 95\% CI, 0.82-1.76), or total mortality (RR, 1.14; 95\% CI, 0.81-1.61). In contrast, cardiovascular events were much higher among patients who maintained less strict BP levels compared with the group on tight $\mathrm{BP}$ control. ${ }^{15}$
CAPPP randomized 10,985 hypertensive patients to either captopril or conventional diuretics or $\beta$-blockers. ${ }^{8}$ BP reduction was equal with both regimes, although the captopril group had a higher $\mathrm{BP}$ at randomization and throughout the study. After 6.1 years, the composite primary endpoint of MI, stroke, or cardiovascular death was not significantly different (RR with captopril 1.05; 95\% CI, 0.90-1.22). Although MI, cardiovascular mortality, total mortality, and cardiac events were all similar, patients using captopril had higher stroke (RR, 1.25 ; $95 \% \mathrm{CI}, 1.01-1.55)$ and lower diabetes (RR, 0.86; 95\% CI, 0.74-0.99). The Swedish Trial in Old Patients with Hypertension 2 recruited 6614 older hypertensive patients and randomized them to conventional therapy (B-blockers or diuretics), calcium channel-blockers (CCBs), or ACEIs. ${ }^{9} \mathrm{Re}-$ duction in $\mathrm{BP}$ was similar in the 3 groups. After 4 to 6 years there was no difference in cardiovascular mortality - the primary endpoint - between conventional therapy (19.8 per 1000 patient-years), ACEIs (20.5 per 1000 patient-years), or CCBs (19.2 per 1000 patient-years). Cardiovascular mortality, MI, stroke, total mortality, diabetes, and heart failure were also equal in these 3 groups.

ALLHAT was the largest clinical hypertensive study ever conducted comparing doxazosin ( $\mathrm{n}=$ 9061), amlodipine ( $\mathrm{n}=9048)$, and lisinopril $(\mathrm{n}=$ 9054) with the diuretic chlorthalidone ( $\mathrm{n}=$ 15,255). ${ }^{11,16}$ The doxazosin arm was terminated early after a median of 3.2 years; systolic BP was approximately $2 \mathrm{~mm} \mathrm{Hg}$ higher with doxazosin. ${ }^{16}$ Although the primary outcome of fatal coronary heart disease and nonfatal MI was equal among patients taking either treatment, the doxazosin arm had more stroke (RR, 1.26; 95\% CI, 1.10-1.46), heart failure (RR, 1.80; 95\% CI, 1.61-2.02), and combined cardiovascular events (RR, 1.20; 95\% CI, 1.13-1.27). The remaining patients had a longer mean follow-up of 4.9 years. ${ }^{11}$ Systolic BP was higher in patients taking amlodipine $(0.8 \mathrm{~mm} \mathrm{Hg}$; $P=.03)$ and lisinopril $(2 \mathrm{~mm} \mathrm{Hg} ; P<.001)$ than in those using chlorthalidone. The 6 -year primary endpoint rate was not significantly different on the diuretic chlorthalidone (11.5\%); CCBs (11.3\%; RR, 0.98 ; 95\% CI, 0.90-1.07), or ACEIs (11.4\%; RR, 0.99; 95\% CI, 0.91-1.08). However, compared with the diuretic, the CCB arm had higher heart failure (RR, 1.38, 95\% CI, 1.25-1.52) whereas the ACEI arm had higher heart failure (RR, 1.19; 95\% 


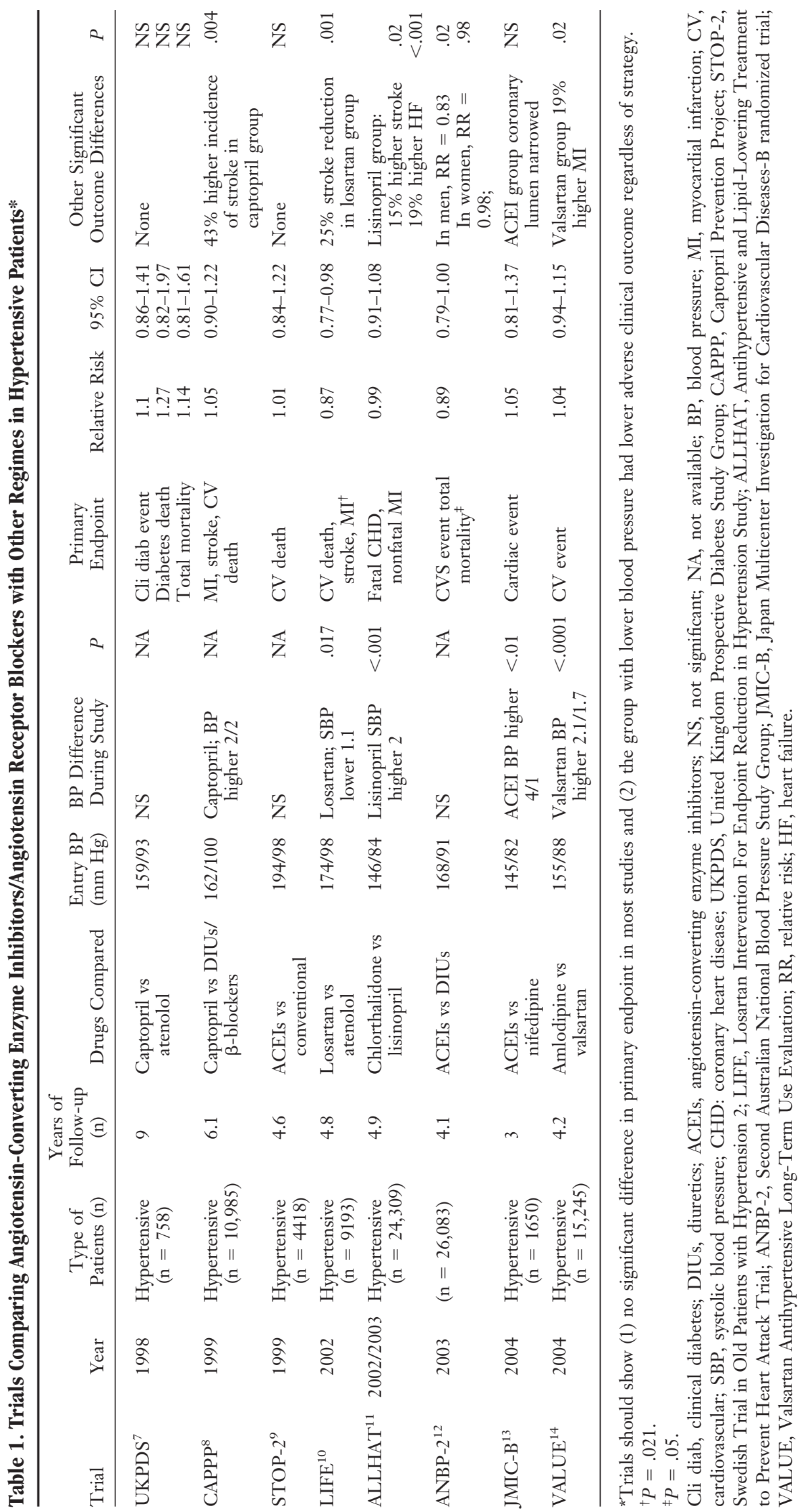


CI, 1.07-1.31); stroke (RR, 1.15; 95\% CI, 1.02$1.30)$; and combined cardiovascular disease (RR, 1.10 ; 95\% CI, 1.05-1.16). Subsequent analysis showed that these findings apply no matter the diabetic state, renal function status, or racial make-up of the patients studied. ${ }^{17-20}$ ALLHAT strongly suggests that lisinopril, an ACEI, has no special cardiovascular protective effect and actually may be inferior to the diuretic in preventing stroke and heart failure. ${ }^{21-24}$

The ANBP2 study was interpreted as showing ACEIs to be better than the diuretic. ${ }^{12}$ Unlike ALLHAT, which was a double-blind trial that followed a strict protocol of therapeutic intervention, ANBP2 was an open-labeled trial of patients randomized to ACEIs $(\mathrm{n}=3044)$ or the diuretic $(\mathrm{n}=$ 3039); the choice of initiating dose and type of drug was left to the participating general practitioner. $\mathrm{BP}$ reduction was similar over 4.1 years and treatment with ACEIs resulted in a lower primary endpoint of cardiovascular events or total death that was of borderline significance (ACEIs, 22.8\% and diuretic, 24.2\%; RR, 0.89; 95\% CI, 0.79-1.00). When considering only the female population (51\% of total), there was no difference between the ACEI and diuretic groups (RR, 1.00; 95\% CI, $0.83-1.22)$. Total mortality, coronary events, heart failure, and stroke were all similar in the 2 groups. Thus, ANBP2 actually confirms the results of ALLHAT by showing that ACEIs and diuretics are almost equivalent in reducing adverse cardiovascular events in hypertensive patients.

The JMIC-B trial randomized 1650 Japanese hypertensive patients with coronary artery disease to either nifedipine-retard or an ACEI. ${ }^{13}$ After 3 years, the primary endpoint comprising cardiac death, MI, angina, heart failure, hospitalization, and coronary intervention was equivalent on nifedipine-retard and ACEI (RR, 1.05; 95\% CI, 0.811.37). Minimum coronary lumen diameter did not change with the use of CCBs but decreased significantly using ACEIs. ${ }^{25}$ Thus, although JMIC-B showed no difference in clinical cardiovascular outcome between CCBs and ACEIs, it raised the possibility of an antiatherogenic effect with $\mathrm{CCB}$ treatment.

The idea that ACEIs may have cardiovascularprotective effects did not come from trials in hypertensive patients but from placebo-controlled trials in patients at high cardiovascular risk. However, these trials have not produced consistent results and interpretation is controversial because patients who were treated always ended up with a lower BP compared with those in the placebo group (Table 2). HOPE recruited 9297 high-risk patients; $80 \%$ had coronary disease, $47 \%$ had hypertension, and $38 \%$ had diabetes. ${ }^{26}$ They were randomized to ramipril $10 \mathrm{mg}$ daily or placebo. After 5 years, the primary endpoint of MI, stroke, or cardiovascular death was significantly reduced with ramipril (14\% vs $17.8 \%$; RR, $0.78,95 \%$ CI, 0.70-0.86). Measured $\mathrm{BP}$ was reduced by $3 / 3 \mathrm{~mm} \mathrm{Hg}$ on ramipril; the HOPE authors argued that this small reduction in BP could not mathematically account for the highly significant cardiovascular outcome reduction. A Swedish HOPE center reported that, although office and daytime BP were only mildly lowered by ramipril, in their cohort the reduction of 24 hour ambulatory BP $(10 / 4 \mathrm{~mm} \mathrm{Hg})$ and nighttime $\mathrm{BP}(17 / 8 \mathrm{~mm} \mathrm{Hg})$ was much larger. ${ }^{32}$ Patients in the HOPE trial were given ramipril at night and office measurement the following day underestimates the actual $\mathrm{BP}$ reduction. A prospective trial using ramipril 1.25 to $10 \mathrm{mg}$ daily among 591 patients produced a mean BP reduction of 20/15 mm Hg over 8 weeks; $85 \%$ of patients having mild to moderate hypertension were successfully controlled using ramipril 2.5 to $5 \mathrm{mg}$ daily in a retrospective review. ${ }^{33,34}$ Thus, it is likely that the $10 \mathrm{mg}$ of ramipril used in the HOPE trial did produce a highly significant BP reduction, in keeping with the Swedish report; this could account for much of the benefit seen.

In EUROPA, 12,218 patients with coronary artery disease $(27 \%$ were hypertensive, $12 \%$ were diabetic) were randomized to perindopril $8 \mathrm{mg}$ daily or matching placebo. ${ }^{28} \mathrm{BP}$ was $9 / 4 \mathrm{~mm} \mathrm{Hg}$ lower in patients in the perindopril treatment arm. After 4.2 years, the primary endpoint of cardiovascular death, MI, or cardiac arrest was $8 \%$ on perindopril and $10 \%$ on placebo $(\mathrm{RR}, 0.8 ; 95 \% \mathrm{CI}$, 0.71-0.91). As in the HOPE study, some have again attributed the clinical benefit with perindopril to the lower BP achieved. ${ }^{35,36}$ In PROGRESS, patients with a previous stroke or transient ischemic attack were randomized to active treatment $(\mathrm{n}=3051)$ or placebo $(\mathrm{n}=3054) .{ }^{27}$ Active treatment was perindopril $4 \mathrm{mg}$, and indapamide was added at the discretion of the doctors. Overall, after a mean of 3.9 years treatment reduced BP by $9 / 4$ 


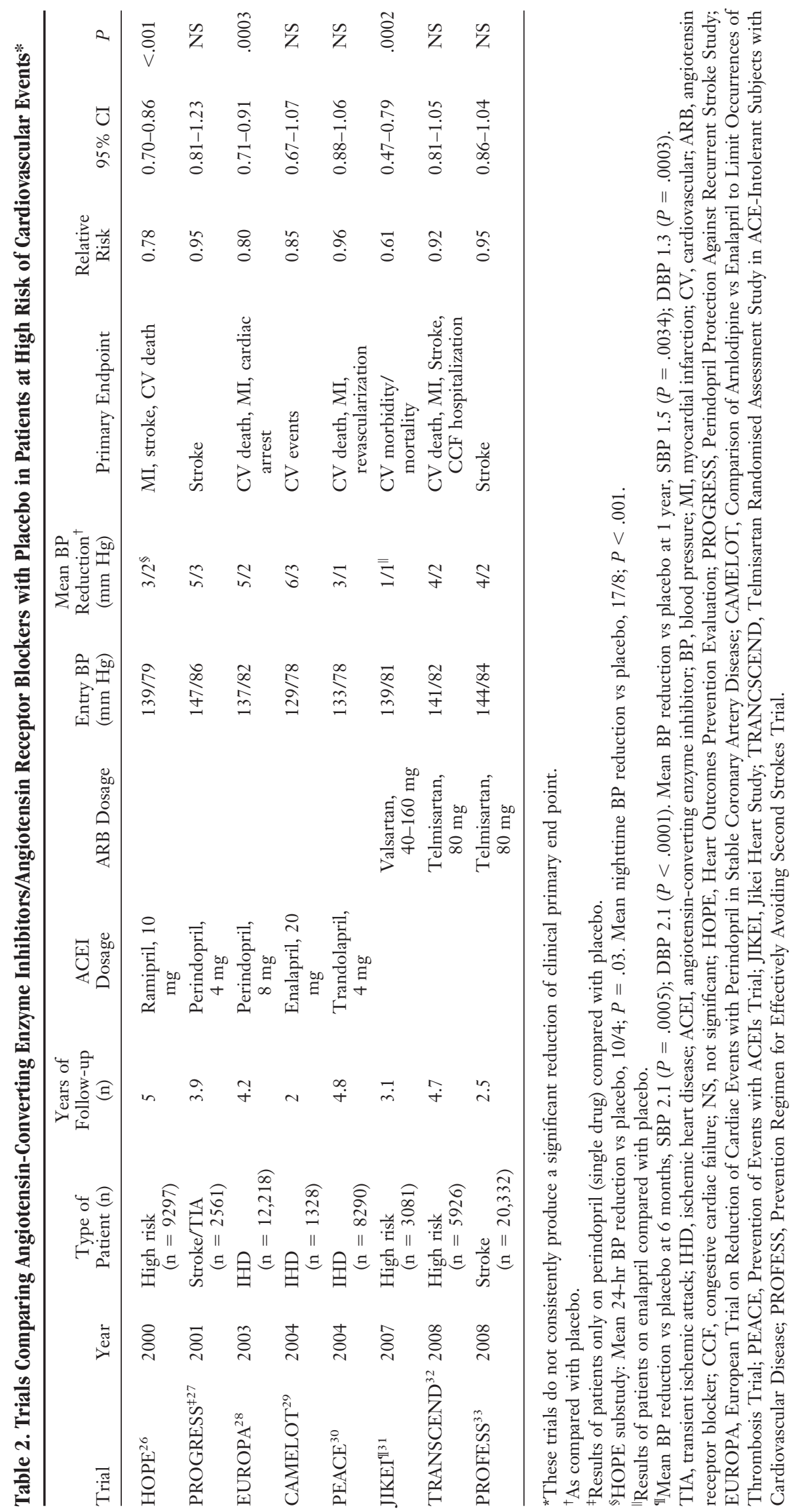


$\mathrm{mm} \mathrm{Hg}$ and produced a significant reduction in the primary endpoint of total stroke (10\% vs $14 \%$; RR, 0.72 ; 95\% CI, 0.62-0.83). Closer analysis of the data showed that patients treated only with the ACEI perindopril had a smaller BP reduction of $5 / 3 \mathrm{~mm} \mathrm{Hg}$ and an insignificant reduction of stroke compared with placebo (RR, 0.95; 95\% CI, 0.771.19). Addition of the diuretic indapamide produced a larger BP reduction $(12 / 5 \mathrm{~mm} \mathrm{Hg})$ and resulted in a highly significant reduction in stroke (RR, 0.52; 95\% CI, 0.46-0.70). Thus, rather than showing that perindopril had special value in stroke reduction, PROGRESS actually showed the value of aggressive $\mathrm{BP}$ reduction in preventing stroke among high-risk patients. ${ }^{37,38}$

Two other trials, PEACE and CAMELOT, also suggest that ACEIs do not have any special cardiovascular protective effects. In PEACE, 8290 patients with stable coronary artery disease and normal left ventricular function were randomized to either trandolapril $4 \mathrm{mg}$ daily or placebo; $45 \%$ had hypertension and $17 \%$ had diabetes. ${ }^{30}$ Mean BP at entry was $133 / 78 \mathrm{~mm} \mathrm{Hg}$; by 36 months it was reduced by 4.4/3.6 mm $\mathrm{Hg}$ on ACEIs and by 1.4/ $2.4 \mathrm{~mm} \mathrm{Hg}$ in the placebo group. After 4.8 years, the primary endpoint of nonfatal MI, cardiovascular death, or revascularization was not significantly different in the 2 groups $(21.9 \%$ trandolapril, $22.5 \%$ on placebo; RR, 0.96 ; $95 \%$ CI, $0.88-1.06$ ). Similarly there was no difference in the individual composite of the primary endpoint or in total mortality. Patients in PEACE were at lower cardiovascular risk than those in EUROPA or HOPE, with a higher proportion using lipid-lowering therapy (entry systolic BP, $139 \mathrm{~mm} \mathrm{Hg}$ [HOPE], $137 \mathrm{~mm}$ $\mathrm{Hg}$ [EUROPA], $133 \mathrm{~mm} \mathrm{Hg}$ [PEACE]; death from cardiovascular causes in placebo, 63\% [HOPE], 59\% [EUROPA], 47\% [PEACE]; lipid-lowering therapy, 29\% [HOPE], 56\% [EUROPA], 72\% [PEACE]). PEACE thus shows that, in low-risk coronary patients who are already on intensive risk factor management, the addition of an ACEI provides no further reduction in clinical cardiovascular outcome.

CAMELOT randomized 1991 high-risk patients with angiographic coronary disease and a diastolic blood pressure below $100 \mathrm{~mm} \mathrm{Hg}$ to placebo, amlodipine, or enalapril. ${ }^{29}$ From a baseline BP of $129 / 78 \mathrm{~mm} \mathrm{Hg}$, during 2 years BP increased 0.7/0.6 $\mathrm{mm} \mathrm{Hg}$ with placebo, decreased 4.8/2.5 $\mathrm{mm} \mathrm{Hg}$ on amlodipine, and decreased 4.9/2.4 mm $\mathrm{Hg}$ on enalapril. The primary cardiovascular endpoint was $23.1 \%$ with placebo, $16.6 \%$ with amlodipine (RR 0.69; 95\% CI, 0.54-0.88) and 20.2\% with enalapril (RR, 0.85; 95\% CI, 0.67-1.07). CAMELOT thus suggests that the CCBs, not ACEIs, may have a special role in reducing cardiovascular clinical events in high-risk patients. Among CAMELOT patients with a systolic BP above the mean, intravascular ultrasound showed significantly less progression of coronary atherosclerosis in patients using amlodipine compared with placebo and no difference in the atherosclerotic progression between enalapril and placebo. As in JMIC-B, CAMELOT suggests it may be the CCBs, not ACEIs, that has a special role in reducing in coronary atheroma.

\section{Do ARBs have Special Cardiovascular Protective Effects?}

As in the case of ACEIs, trials assessing the role of $\mathrm{ARBs}$ in cardiovascular disease prevention can be divided into 2 groups: those comparing ARBs with other drugs in hypertensive patients and those comparing ARBs with placebos in patients at high cardiovascular risk (Tables 1 and 2).

LIFE randomized 9193 hypertensive patients with left ventricular hypertrophy to either losartan or atenolol. ${ }^{10}$ There was a marked reduction of stroke in the losatan group (5\% vs 7\%; RR, 0.87; 95\% CI, 0.63-0.89), and this caused a significant reduction in the composite primary endpoint of death, MI, or stroke (11\% vs $13 \%$; RR, 0.87 ; $95 \%$ CI, 0.77-0.98). New-onset diabetes was lower in patients using losartan, suggesting that angiotensin antagonism has a favorable effect on glucose metabolism, a finding also noted with captopril in the CAPPP trial. When reviewing only the 1195 diabetic patients in LIFE, ${ }^{39}$ the benefit of losatan was more remarkable, with a significant reduction not only in the primary endpoint (RR, 0.76; 95\% CI, $0.58-0.98)$, but also in cardiovascular and total mortality. Surprisingly, stroke reduction with losartan was not statistically significant in this diabetic population (RR, 0.79; 95\% CI, 0.55-1.14). The results of LIFE should be read together with data from other trials. ACEIs were weaker than the comparator drug in preventing stroke in both CAPPP and ALLHAT., ${ }^{8,11}$ Only approximately $10 \%$ of patients were on monotherapy with losar- 
$\tan$ or atenolol at the end of the LIFE trial; thus it is misleading to solely attribute the better clinical outcome to the superiority of losartan over atenolol when so few patients were actually on monotherapy. ${ }^{40}$ The mean systolic BP was lower in patients on losartan in both the overall $(1.1 \mathrm{~mm} \mathrm{Hg}$; $P=.017)$ and diabetic $(2 \mathrm{~mm} \mathrm{Hg} ; P$ not stated $)$ patients, making it possible that the benefit resulted from better BP reduction on the losartan arm. Furthermore, recent reports suggest that $\beta$-blockers, especially atenolol, may be less useful in older hypertensive patients and are specially weak in preventing stroke. ${ }^{41-43}$ LIFE actually shows the importance of $\mathrm{BP}$ reduction and the weaker efficacy of atenolol in stroke prevention, not a special cardiovascular protective effect with losartan.

The VALUE trial randomized 15,245 hypertensive patients to either valsartan or amlodipine. ${ }^{14,44}$ Attained BP was significantly lower with amlodipine. After 4.2 years there was no difference in the primary endpoint of first cardiac event (RR, 1.04; 95\% CI, 0.94-1.15). Although the occurrence of new diabetes was lower among patients using valsartan (RR, 0.77; 95\% CI, 0.69-0.86), incidence of MI was higher among patients using the ARB (RR, 1.19; 95\% CI, 1.02-1.38). However, after correction for the BP difference, $\mathrm{MI}$ incidence was similar in the 2 groups (RR, 0.97; 95\% CI, 0.80-1.19). ${ }^{45}$ Patients reaching adequate BP control by 6 months were shown to fare better, regardless of drug type used. The benefit of good BP control is thus more important than the subtle differences between antihypertensive drugs. A better metabolic profile in the ARB arm did not translate into a reduction in adverse outcome. The VALUE trial, as does ALLHAT, suggests that drugs targeting the rennin-angiotensin system do not have special cardiovascular protective effects despite producing a better metabolic profile; they also seem less efficacious in reducing $\mathrm{BP}$ compared with CCBs and diuretics. $^{16}$

The JIKEI Heart Study randomized 3081 highrisk patients with cardiovascular disease to valsartan 40 to $160 \mathrm{mg}$ daily or other treatment that excludes ARBs. ${ }^{31}$ After 3.1 years, the primary endpoint of cardiovascular mortality and morbidity was significantly lower with valsartan (RR, 0.61; 95\% CI, $0.47-0.79)$, driven by lower stroke and transient ischemic attack (RR, 0.60; 95\% CI, 0.38-0.95), angina (RR, 0.35; 95\% CI, 0.20-0.58), and heart failure (RR, 0.53; 95\% CI, 0.31-0.94). Although mean initial $(139 / 81 \mathrm{~mm} \mathrm{Hg})$ and final $(132 / 77 \mathrm{~mm}$ $\mathrm{Hg}) \mathrm{BPs}$ were reported to be similar to those in patients in the valsartan and placebo groups, at 6 months $\mathrm{BP}$ was $2 / 2 \mathrm{~mm} \mathrm{Hg}$ lower in the patients treated with the ARB. In the VALUE trial, patients reaching a lower BP at 6 months had fewer clinical events, regardless of the antihypertensive drug used. ${ }^{45}$ It is thus debatable whether the reduction in clinical outcome in the JIKEI study is because of a beneficial effect of valsartan or the better BP levels achieved. ${ }^{46}$

The Telmisartan Randomised Assessment Study in ACE-Intolerant Subjects with Cardiovascular Disease (TRANSCEND) trial randomized 5926 patients intolerant of ACEIs who had prior cardiovascular disease or diabetes with end-organ damage to either telmisartan $80 \mathrm{mg}$ daily or placebo. ${ }^{47}$ Mean follow-up was 56 months. Although BP was lower by $4.0 / 2.2 \mathrm{~mm} \mathrm{Hg}$ in the telmisartan group, the primary composite outcome (cardiac death, MI, stroke, or hospitalization for heart failure) was similar in the 2 groups $(15.7 \%$ telmisartan, $17 \%$ placebo; RR, 0.92; 95\% CI, 0.81-1.05). The reduction in secondary outcome (cardiac death, $\mathrm{MI}$, or stroke) was of borderline significance (RR, 0.87; $95 \% \mathrm{CI}$, 0.76-1.00). TRANSCEND thus does not strongly suggest a cardiovascular protective role for the ARBs. In the Prevention Regimen for Effectively Avoiding Second Strokes Trial (PROFESS), 20,332 patients who had recently had an ischemic stroke were randomized to either telmisartan 80 $\mathrm{mg}$ or placebo and followed for a mean of 2.5 years. ${ }^{48}$ Although the BP was 3.8/2.0 $\mathrm{mm} \mathrm{Hg}$ lower in the telmisartan group, the primary endpoint of recurrent stroke was not significantly reduced (8.7\% telmisartan, 9.2\% placebo; RR, 0.95; 95\% CI, 0.86-1.04). Major cardiovascular events (RR, 0.94; 95\% CI, 0.87-1.01), and new diabetes (RR, $0.82 ; 95 \%$ CI, $0.65-1.04)$ were also not significantly altered. It is interesting to compare how strikingly similar the results of PROFESS are to those of PROGRESS. ${ }^{27}$ In PROGRESS, among patients only on perindopril the $\mathrm{BP}$ reduction was $5 / 3 \mathrm{~mm} \mathrm{Hg}$, with reduction of stroke (RR, 0.95) and major vascular event (RR, 0.96) not significantly different from placebo. In PROFESS, the $\mathrm{BP}$ reduction of $3.8 / 2.0 \mathrm{~mm} \mathrm{Hg}$ produced a nonsignificant reduction of stroke (RR, 0.95) and cardiovascular event (RR, 0.94). Thus, PROFESS 
Table 3. Discontinuation Rate of Antihypertensive Drugs in the Comparative Trials

\begin{tabular}{lccccc}
\hline & \multicolumn{5}{c}{ Antihypertensive Drug Class (\%) } \\
\cline { 2 - 6 } Trial & ACEI & ARB & ACEI + ARB & BB & CCB \\
\hline ONTARGET $(\mathrm{n}=25,620)$ & 25 & 23 & 29 & DIU \\
LIFE $(\mathrm{n}=9193)$ & & 23 & 26 & 24 \\
VALUE $(\mathrm{n}=15,245)$ & 27 & & 35 & 20 \\
ALLHAT $(\mathrm{n}=24,309)$ & 22 & & & 5 \\
UKPDS $(\mathrm{n}=758)$ & 9 & & & 5 \\
JMIC-B $(\mathrm{n}=1650)$ & & & & \\
\hline
\end{tabular}

ACEI, angiotensin-converting enzyme inhibitor; ARB, angiotensin receptor blocker; CCB, calcium channel blocker; DIU, diuretic; BB, $\beta$-blocker; ONTARGET, Ongoing Telmisartan Alone and in Combination with Ramipril Global Endpoint Trial; LIFE, Losartan Intervention For Endpoint Reduction in Hypertension Study; VALUE, Valsartan Antihypertensive Long-Term Use Evaluation; ALLHAT, Antihypertensive and Lipid-Lowering Treatment to Prevent Heart Attack Trial; UKPDS, United Kingdom Prospective Diabetes Study Group; JMIC-B, Japan Multicenter Investigation for Cardiovascular Diseases-B randomized trial.

confirms the impression of PROGRESS that the rennin-angiotensin antagonists do not have a special stroke-reducing or cardiovascular-preventive effect.

\section{What is the Lesson from ONTARGET?}

The ONTARGET trial sought to answer 2 questions: whether ARBs are similar to ACEIs in therapeutic efficacy and whether their combination could produce even better clinical results. ${ }^{49} \mathrm{~Pa}-$ tients with vascular disease or diabetes were randomized to $10 \mathrm{mg}$ ramipril $(\mathrm{n}=8576), 80 \mathrm{mg}$ telmisartan $(\mathrm{n}=8542)$, or both $(\mathrm{n}=8502)$. From the same initial level of $142 / 82 \mathrm{~mm} \mathrm{Hg}$, after 6 weeks $\mathrm{BP}$ fell to $135 / 78 \mathrm{~mm} \mathrm{Hg}$ on ramipril, 134/77 mm Hg on telmisartan, and 132/76 mm Hg on combination therapy. The primary endpoint was a composite of cardiovascular death, MI, stroke, or hospitalization for heart failure. After a median of 56 months, compared with ramipril there was no difference in the primary endpoint with telmisartan (RR, 1.01; 95\% CI, 0.94-1.09) or combination therapy (RR, 0.99; 95\% CI, 0.921.07). ONTARGET thus showed that ARBs and ACEIs are equivalent in their clinical efficacy. The absence of any reduction in cardiovascular outcome from the ACEI and ARB combination arm is also another piece of evidence suggesting that antagonizing the rennin-angiotensin system does not produce any special cardiovascular-protective effect.

Although adverse effects and discontinuation rates were lower with telmisartan compared with ramipril, the discontinuation rate with telmisartan of $23 \%$ is a reminder that ARBs are not free of adverse effects. In fact, reviewing the discontinua- tion rates in drug trials of hypertensive patients suggest that the CCBs and diuretics actually may be best when seeking to lower discontinuation and enhance compliance (Table 3). Although the combination of ramipril and telmisartan better reduced proteinuria compared with ramipril alone, major renal outcomes (need for dialysis, doubling of serum creatinine, and death) were surprising higher in the combination group ( $14.5 \%$ vs $13.5 \%$; RR, 1.09; 95\% CI, 1.01-1.18). ${ }^{50}$ Furthermore, adverse side effects were highest with combination therapy. Thus, it may be that patients respond best when different strategies are used for treatment, and excessively targeting a single pathway will result in less clinical benefit with higher risk of adverse consequences.

\section{Conclusion}

It is hard to escape the conclusion that the renninangiotensin antagonists do not show special cardiovascular-protective properties and that there are no major differences between the different antihypertensive drugs in their ability to reduce adverse cardiovascular outcomes. ${ }^{51}$ There was no significant difference in the cardiovascular primary endpoint in most of the comparative drug trials done among hypertensive patients (Table 1). In CAPPP (captopril vs $\beta$-blocker/diuretic); ALLHAT (doxazosin, amlodipine, lisinopril vs chlorthalidone); LIFE (losartan vs atenolol); and VALUE (amlodipine vs valsartan), where a major cardiovascular endpoint was reduced in one of the treatment arms, it was always the arm with the lower achieved BP that saw the better clinical outcome..$^{8,10,11,14,16,39}$ Thus, in- 
stead of trying to work out why the angiotensin antagonists could be cardioprotective in some trials (LIFE) but not in others (CAPPP, ALLHAT, VALUE), the simple and consistent message is that the lower the achieved BP the lower the adverse cardiovascular outcome in hypertensive patients.

The absence of a cardioprotective effect with the angiotensin antagonist is reinforced by the inconsistent results of trials comparing ACEIs and ARBs with placebo in patients at high risk for cardiovascular events (Table 2). HOPE, EUROPA, and the JIKEI Heart Study showed significant reduction of cardiac outcomes, whereas PROGRESS, PEACE, CAMELOT, TRANSCEND, and PROFESS did not. $^{26-31,47,48}$ In CAMELOT, compared with the placebo group cardiovascular outcome was not affected by enalapril but was significantly lower in patients using amlodipine. ${ }^{29}$ Furthermore, progression of coronary atherosclerosis was retarded with amlodipine but showed no difference between the enalapril and placebo groups. Thus rather than the ACEIs, it may be the CCBs that have an antiatherosclerotic effect.

The case against the angiotensin antagonists having a special cardiovascular protective effect is strongly supported by the results from ONTARGET. This study showed that combining ARBs with ACEIs, each at half of the maximal doses, resulted in a lower BP, did not lower cardiovascular outcome, and produced a higher incidence of renal and other adverse events. ${ }^{49,50}$ If ACEIs and ARBs are especially useful in protecting against cardiac disease, then logically the combination of ARBs with ACEIs should further lower cardiovascular outcomes. This absence of a reduction in cardiovascular outcome from the ACEI and ARB combination arm in ONTARGET is further evidence that suggests that these drugs do not have any special cardiovascular-protective effect.

The higher incidence of adverse events with combination therapy in ONTARGET emphasizes the point that ARBs and ACEIs are not free of side effects (Table 3). In ALLHAT, the discontinuation rate of patients using ACEIs was higher than that in those using a diuretic, whereas in VALUE the incidence of dizziness, headache, angina, diarrhea, and syncope were all significantly higher in patients using ARBs compared with those using CCBs. ${ }^{11,14}$ Clinicians initiating patients onto ARB or ACEI treatment must be aware of these potential adverse effects, which may reduce compliance during longterm maintenance therapy.

Finally, it is interesting to consider what target $\mathrm{BP}$ to aim for when seeking to reduce cardiovascular outcomes in high-risk patients. In PEACE, the $\mathrm{BP}$ at trial initiation was $135 / 78 \mathrm{~mm} \mathrm{Hg}$; treatment with trandolapril lowered BP but did not reduce cardiovascular events. ${ }^{30}$ In ONTARGET, the ramipril group was treated to a $\mathrm{BP}$ of $133 / 78 \mathrm{~mm}$ $\mathrm{Hg}$; telmisartan or combination therapy lowered BP further but did not further reduce clinical cardiovascular outcomes. ${ }^{49}$ Thus, 135/80 mm Hg represents a reasonable target when seeking to reduce cardiovascular outcomes. The hypertension guidelines all define normal $\mathrm{BP}$ to be $<135 / 80 \mathrm{~mm} \mathrm{Hg}$, although optimal BP is said to be $<120 / 80 \mathrm{~mm}$ Hg. ${ }^{52-55}$ Although epidemiologic reviews suggest that the lowest risk of cardiovascular disease occur at a systolic BP of $<120 \mathrm{~mm} \mathrm{Hg}$, further clinical trials are need to decide if pharmacological treatment to below the normal BP of $135 / 80 \mathrm{~mm} \mathrm{Hg}$ produces clinical benefit. ${ }^{56-59}$ It is important to also remember that all cardiovascular risk factors require appropriate management. ${ }^{60}$ Just as $\mathrm{ON}$ TARGET showed that increasingly attacking the angiotensin pathway did not bring increasing clinical benefit, when seeking to reduce cardiovascular outcome it may be more fruitful to target different pathologic processes and risk factors instead of just concentrating on reducing BP levels. ${ }^{61}$

\section{Treatment Recommendations}

(1) For hypertensive patients, ACEIs and ARBs are equivalent but not superior to other antihypertensive drugs in their cardiovascular protective effects (GRADE A), and clinical cardiovascular outcomes will be reduced with tight $\mathrm{BP}$ control (GRADE A).

(2) For patients with normal left ventricular function who are at high risk of cardiovascular events, ACEIs and ARBs do not exert special cardiovascular-protective effects additive to their antihypertensive action (GRADE B), and clinical cardiovascular outcome is best reduced by treatment of all risk factors, including normalizing BP levels (to $<135 / 80 \mathrm{~mm} \mathrm{Hg}$ ) and not by adding ACEIs/ ARBs to every patient's treatment (GRADE B).

\section{References}

1. Sleight P, Yusuf S. New evidence on the importance of the rennin-angiotensin system in the treatment of 
higher-risk patients with hypertension. J Hypertens 2003;21:1599-608.

2. Weir MR. Effects of rennin-angiotensin system inhibition on end-organ protection: can we do better? Clin Ther 2007;29:1803-24.

3. Stojiljkovic L, Behnia R. Role of rennin angiotensin system inhibitors in cardiovascular and renal protection: a lesson from clinical trials. Curr Pharm Des 2007;13:1335-445.

4. Julius S, Nesbitt SD, Egan BM, et al. Feasibility of treating prehypertension with an angiotensin-receptor blocker. N Engl J Med 2006;354:1685-97.

5. The Action to Control Cardiovascular Risk in Diabetes (ACCORD) study group. Effects of intensive glucose lowering in type 2 diabetes. N Engl J Med 2008;358: 2545-59.

6. Barter PJ, Caulfield M, Eriksson M, et al. Effects of torcetrapid in patients at high risk of coronary events. N Engl J Med 2007;357:2109-22.

7. UK Prospective Diabetes Study Group. Efficacy of atenolol and captopril in reducing risk of macrovascular and microvascular complications in type 2 diabetes: UKPDS 39. BMJ 1998;317:713-20.

8. Hansson L, Lindholm LH, Niskanen L, et al. Effect of ACE inhibition compared with conventional therapy on cardiovascular morbidity and mortality in hypertension: The Captopril Prevention Project (CAPPP). Lancet 1999;353:611-6.

9. Hansson L, Lindholm LH, Ekbom T, et al. Randomized trial of old and new antihypertensive drugs in elderly patients: cardiovascular mortality and morbidity the Swedish Trial in Old Patients with Hypertension 2 (STOP- Hypertension 2) study. Lancet 1999;354:1751-6.

10. Dahlof B, Devereux RB, Kjeldsen SE, et al. Cardiovascular morbidity and mortality in the losartan Intervention For Endpoint reduction in hypertension study (LIFE): a randomised trial against atenolol. Lancet 2002;359:995-1003.

11. The ALLHAT Officers and Coordinators for the ALLHAT Collaborative Research Group. Major outcomes in high-risk hypertensive patients randomized to angiotensin-converting enzyme inhibitor or calcium channel blocker vs diuretic: the Antihypertensive and Lipid-Lowering Treatment to Prevent Heart Attack Trial (ALLHAT). JAMA 2002;288: 2981-97.

12. Wing $\mathrm{LMH}$, Reid CM, Ryan $\mathrm{P}$, et al. A comparison of outcomes with angiotensin coverting enzyme inhibitors and diuretics for hypertension in the elderly. N Engl J Med 2003;348:583-92.

13. Yui Y, Sumiyoshi T, Kodama K, et al. Comparison of nifedipine retard with angiotensin-converting enzyme inhibitors in Japanese hypertensive patients with coronary artery disease: the Japan Multicenter Investigation for Cardiovascular Diseases-B (JMIC-B) randomized trial. Hypertens Res 2004;27:181-91.

14. Julius S, Kjeldsen SE, Weber M, et al. Outcomes in hypertensive patients at high cardiovascular risk treated with regimens based on valsartan or amlodipine: the VALUE randomized trial. Lancet 2004; 363:2022-31.

15. UK Prospective Diabetes Study Group. Tight blood pressure control and risk of macrovascular and microvascular complications in type 2 diabetes: UKPDS 38. BMJ 1998;317:703-13.

16. ALLHAT Officers and Coordinators for the ALLHAT Collaborative Research Group. Diuretic versus alpha-blocker as first-step antihypertensive therapy: final results from the Antihypertesnive and Lipid-Lowering Treatment to Prevent Heart Attack Trial (ALLHAT). Hypertension 2003;42:239-46.

17. Barzilay JI, Davis BR, Bettencourt J, et al. Cardiovascular outcomes using doxazosin vs chlorthalidone for the treatment of hypertension in older adults with and without glucose disorders: a report from the ALLHAT Study. J Clin Hypertens 2004;6:11625.

18. Whelton PK, Barzilay J, Cushman WC, et al. Clinical outcomes in antihypertensive treatment of type 2 diabetes, impaired fasting glucose concentration, and normoglycemia. Antihypertensive and Lipid-Lowering Treatment to Prevent Heart Attack Trial (ALLHAT). Arch Intern Med 2005;165:1401-9.

19. Rahman M, Pressel S, Davis BR, et al. Renal outcomes in high-risk hypertensive patients treated with an angiotensin-converting enzyme inhibitor or a calcium channel blocker vs a diuretic: a report from the ALLHAT Study. Arch Inten Med 2005;165:936-46.

20. Wright JT, Dunn JK, Cutler JA, et al. Outcomes in hypertensive black and nonblack patients treated with chlorthalidone, amlodipine, and lisinopril. JAMA 2005;293:1595-607.

21. Williams B. Drug treatment for hypertension: most patients will need a treatment cocktail-including a thiazide diuretic. BMJ 2003;326:61-2.

22. Moser M. Results of ALLHAT. Is this the final answer regarding initial anti-hypertensive drug therapy? Arch Intern Med 2003;163:1269-73.

23. Appel LJ. The verdict from ALLHAT-Thiazide diuretics are the preferred initial therapy for hypertension. JAMA 2002;288:3039-42.

24. Davis BR, Furberg CD, Wright JT Jr, Cutler JA, Whelton P. ALLHAT: setting the record straight. Ann Intern Med 2004;141:39-46.

25. Shinoda E, Yui Y, Kodama K, et al. Japan Multicenter Investigation for Cardiovascular Diseases-B Study Group. Quantitative coronary angiogram analysis: nifedipine retard versus angiotensin-converting enzyme inhibitors (JMIC-B side arm study). Hypertension 2005;45:1153-8.

26. The Heart Outcomes Prevention Evaluation Study Investigators. Effects of an angiotensin-converting enzyme inhibitor, ramipril, on cardiovascular events in high-risk patients. N Engl J Med 2000;342:14553. 
27. PROGRESS collaborative group. Randomised trial of a perindopril-based blood pressure lowering regimen among 6105 individuals with previous stroke or transient ischemic attack. Lancet 2001;358:1033-41.

28. The EURopean trial On reduction of cardiac events with Perindopril in stable coronary Artery disease Investigators. Efficacy of perindopril in reduction of cardiovascular events among patients with stable coronary artery disease: randomized double-blind, placebo-controlled, multicentre trial (the EUROPA study). Lancet 2003;362:782-88.

29. Nissen SE, Tuzcu EM, Libby P, et al. Effect of antihypertensive agents on cardiovascular events in patients with coronary disease and normal blood pressure. The CAMELOT Study: a randomized controlled trial. JAMA 2004;292:2217-26.

30. The PEACE Trial Investigators. Angiotensin-converting enzyme inhibition in stable coronary artery disease. N Engl J Med 2004;351:2058-68.

31. Mochizuki S, Dahlof B, Shimizu M, et al. Valsartan in a Japanese population with hypertension and other cardiovascular disease (Jikei Heart Study): a randomized, open-label, blinded end-point morbidity-mortality study. Lancet 2007;369:1431-9.

32. Svensson P, de Faire U, Sleight P, Yusuf S, Ostergren J. Comparative effects of ramipril on ambulatory and office blood pressures: a HOPE substudy. Hypertension 2001;38:28-32.

33. Kaplan NM, Sproul LE, Mulcahy WS. Large prospective study of ramipril in patients with hypertension. CARE Investigators. Clin Ther 1993;15: 810-8.

34. Frampton JE, Peters DH. Ramipril. An updated review of its therapeutic use in essential hypertension and heart failure. Drugs 1995;49:440-66.

35. Moutsatsos GD. More hype than HOPE. Hypertension 2003;41:e4.

36. Kwaku M, Burman KD, Becker KL, Mannan M. The EUROPA trial. Lancet 2003;362:1935.

37. Wennberg R, Zimmermann C. The PROGRESS trial three years later: time for a balanced report of effectiveness. BMJ 2004;329:968-70.

38. MacMahon S, Neal B, Rodgers A, Chalmers J. The PROGRESS trial three years later: time for more action, less distraction. BMJ 2004;329:970-1.

39. Lindholm LH, Ibsen H, Dahlof B, et al. Cardiovascular morbidity and mortality in patients with diabetes in the Losartan Intervention For Endpoint reduction in hypertension study (LIFE): a randomized trial against atenolol. Lancet 2002;359:100410.

40. Ong HT. LIFE: lorsatan versus atenolol. Lancet 2003;362:1416.

41. Carlberg B, Samuelsson O, Lindholm LH. Atenolol in hypertension: is it a wise choice? Lancet 2004;364: 1684-9.

42. Khan N, McAlister FA. Re-examining the efficacy of beta-blockers for the treatment of hypertension: a meta-analysis. CMAJ 2006;174:1737-42.

43. Lindholm LH, Carlberg B, Samuelsson O. Should beta-blockers remain first choice in the treatment of primary hypertension? A meta-analysis. Lancet 2005;366:1545-53.

44. Kjeldsen SE, Julius S, Brunner H, et al. Characteristics of 15314 hypertensive patients at high coronary risk. The VALUE Trial. Blood Press 2001;10:83-91.

45. Weber MA, Julius S, Kjeldsen SE, et al. Blood pressure dependent and independent effects of antihypertensive treatment on clinical events in the VALUE Trial. Lancet 2004;363:2049-51.

46. Staessen JA, Richart T. Sum and substance in the Jikei heart study. Lancet 2007;369:1407-8.

47. The Telmisartan Randomised Assessment Study in ACE-Intolerant Subjects with Cardiovascular Disease (TRANSCEND) investigators. Effects of the angiotensin-receptor blocker telmisartan on cardiovascular events in high-risk patients intolerant to angiotensin-converting enzyme inhibitors: a randomized controlled trial. Lancet 2008;372:1174-83.

48. Yusuf S, Diener HC, Sacco RL, et al. Telmisartan to prevent recurrent stroke and cardiovascular events. N Engl J Med 2008;359:1225-37.

49. The ONTARGET Investigators. Telmisartan, ramipril or both in patients at high risk for vascular events. N Engl J Med 2008;358:1547-59.

50. Mann JFE, Schmeider RE, McQueen M, et al. Renal outcomes with telmisartan, ramipril, or both in people at high vascular risk (the ONTARGET study): a multicentre, randomized, double-blind, controlled trial. Lancet 2008;372:547-53.

51. Ong HT. Cardiovascular outcomes in the comparative hypertension drug trials: more consensus than controversy. Singapore Med J 2008;49:599-606.

52. Chobanian AV, Bakris GL, Black HR, et al. The Seventh Report of the Joint National Committee on Prevention, Detection, Evaluation, and Treatment of High Blood Pressure. The JNC 7 Report. JAMA 2003;289:2560-72.

53. Whitworth JA, World Health Organization, International Society of Hypertension Writing Group. 2003 World Health Organization (WHO)/International Society of Hypertension (ISH) statement on management of hypertension. J Hypertens 2003;21: 1983-92.

54. Williams B, Poulter NR, Brown MJ, et al. Guidelines for management of hypertension: report of the fourth working party of the British Hypertension Society, 2004-BHS IV. J Hum Hypertens 2004;18: 139-85

55. Mancia G, de Backer G, Dominiczak A, et al. 2007 Guidelines for the management of arterial hypertension. Eur Heart J 2007;28:1462-536.

56. Pepine CJ. What is the optimal blood pressure and drug therapy for patients with coronary artery disease? JAMA 2004;292:2271-3. 
57. Prospective Studies Collaboration. Age-specific relevance of usual blood pressure to vascular mortality: a meta-analysis of individual data for one million adults in 61 prospective studies. Lancet 2002;360: 1903-13.

58. Vasan RS, Larson MG, Leip EP, et al. Impact of high-normal blood pressure on the risk of cardiovascular disease. N Engl J Med 2001;345:1291-7.

59. Alder AI, Stratton IM, Neil HA, et al. Association of systolic blood pressure with macrovascular and microvascular complications of type 2 diabetes (UKPDS 36): prospective observational study. BMJ 2000;321:412-9.

60. Daviglus ML, Liu K. Today's Agenda. We must focus on achieving favorable levels of all risk factors simultaneously. Arch Intern Med 2004;164:2086-7.

61. Pitt B. ACE Inhibitors for patients with vascular disease without left ventricular dysfunction-may they rest in PEACE? N Engl J Med 2004;351: 2115-7. 